\title{
HIGH SENSITIVITY BIDIRECTIONAL PRESSURE SENSOR BASED ON FORCE FREQUENCY EFFECT IN MICROMACHINED AT-CUT QUARTZ RESONATORS
}

Nishit Goel ${ }^{1}$, Stephen Bart ${ }^{2}$ and Srinivas Tadigadapa ${ }^{l, 3}$

${ }^{1}$ Department of Electrical Engineering, ${ }^{3}$ Materials Research Institute, The Pennsylvania State University, University Park, Pennsylvania, 16802, USA.

${ }^{2}$ MKS Instruments Inc., 6 Shattuck Road, Andover, MA 01810, USA.

\begin{abstract}
In this paper, we report an AT-cut quartz diaphragm pressure sensor based on force frequency effect. Commercially available $100 \mu \mathrm{m}$ thick AT-cut quartz blanks were thinned down using micromachining processes to achieve high pressure sensitivity. The quartz sensor is resonated in thickness shear mode (TSM) and change in resonance frequency is monitored with change in pressure. Based on the signal-to-noise performance, we report a resolution of $2.39 \mathrm{mT}$ Torr for the $83.56 \mathrm{MHz}$ resonator and 11.14 mTorr for the $45.5 \mathrm{MHz}$ resonator. The range of linear operation for $45.5 \mathrm{MHz}$ and $83.56 \mathrm{MHz}$ was comparable to up to 80 Torr with the latter having higher sensitivity.
\end{abstract}

\section{INTRODUCTION}

Vacuum pressure sensors are widely used by industries like semiconductor equipment manufacturing and biomedical instrumentation in systems like metal deposition, plasma processing, process control, etc. These processes require a high vacuum environment and thus require sensors that can sense pressures in the range of $10^{3}$ Torr to $10^{-8}$ Torr. Since there is no single technology that can operate throughout this regime, a combination of multiple pressure sensors, like Pirani gage (for $\sim 760-10^{-3}$ Torr) and cold cathode gage (for $\sim 10^{-2}-10^{-8}$ Torr), is employed.

Micro electro mechanical systems (MEMS) based vacuum sensors have been extensively studied, but so far have been limited by the range of operation, reliability in various environments or bulkier integration with systems [1]. Micromachined Pirani gages are available commercially, but they suffer from small range of operation ( 10 - 100mTorr) [2]-[4]. Miniaturized ionization gages are good for the high vacuum regime but are not suitable for pressures $>1$ mbar absolute pressure [5]-[7].

Both Pirani and cold cathode sensors rely on indirect inference of pressure based upon heat transfer and ionization currents that depend not only on the pressure but also on the gas composition and various other factors relating to a vacuum chamber. Capacitance diaphragm gages $(\mathrm{CDG})$ measure pressure by sensing the deflection of a diaphragm to applied pressure. These devices can provide highly accurate measurements of vacuum from $10^{3}-10^{-3}$ Torr. However, several of these sensors with varying full scale ranges are required to cover such a large pressure range with optimized resolution. Further extension of the range of the CDGs is extremely challenging since the change in capacitance for pressure signals of less than $10^{-4}$ Torr results in extremely small diaphragm deflections. Under such inputs, the corresponding capacitances changes are in the $10^{-15}-10^{-18} \mathrm{~F}$ range and therefore difficult to resolve under standard operating ambient. Extensive research was done on micromachined pressure sensors in the early 1990's showing the potential of such devices for high vacuum measurements [8]-[10].

Force frequency effect in quartz crystal has been known since the 1960's, where researchers were trying to understand the stability of crystal oscillators under various applied load conditions [11]. Specifically, the case of response of a quartz plate resonator under transverse loading conditions was theoretically studied by Markenscoff and Lee [12]. The fractional frequency change $\Delta f / f_{0}$ was determined to be a function of the zeroth and first order strains and first order strain gradients, and is related through second and third order elastic stiffness. This effect is used extensively for pressure sensing using quartz resonators in oil exploration for pressure measurements $>1$ bar [13]. However, due to the lack of effective micromachining techniques for quartz, the use of this effect for sensing vacuum pressures has not been explored.

With the motivation to span a large pressure range with high resolution, we present a micromachined AT-cut quartz diaphragm pressure sensor utilizing the force frequency effect. Unlike a CDG, the quartz pressure sensor does not require a closely placed (sub-micron) counter electrode and hence places less stringent constraints in the pressure sensor design. Thinned inverted mesa quartz resonators can be readily fabricated using deep reactive ion etching (DRIE) of quartz. Differential pressure applied across such a high- $Q$ thickness shear mode resonator diaphragm results in a uniform transverse load and consequently bending of the quartz diaphragm. The resulting change in resonance frequency is monitored by constantly measuring the at-resonance impedance characteristics of the resonator as function of applied pressure. This design allows for the realization of differential, gage, or absolute pressure sensing depending upon the pressure used on one of the faces (reference pressure) of the diaphragm. Sensitivity and range of operation with different thickness of resonators has also been studied. We also observed bi-directionality in the response of the device.

\section{THEORETICAL BACKGROUND}

Application of a differential pressure across the quartz diaphragm of circular shape will result in a peak deflection $w_{0}$ at the center of the diaphragm. In the absence of any residual stress in the diaphragm, the peak deflection of the quartz diaphragm can be related to the differential pressure $\Delta p$ as [14]

$$
w_{0}=\left(\frac{3}{8} \frac{r_{q}^{4}}{t_{q}} \frac{1-v_{q}^{2}}{E_{q}} \Delta p\right)^{1 / 3}
$$

where $t_{q}$ is the thickness and $r_{q}$ is the radius of the quartz resonator diaphragm, $E_{q}$ is the Young's modulus and $v_{q}$ is the Poisson's ratio of quartz respectively.

For a rectangular plate resonator, clamped on one end and with an applied point load at the free end of the beam, the model for frequency shift of the quartz resonator with applied load in terms of second and third order elastic constants and zeroth and first order strains can be given by [12]:

$$
\frac{\Delta f}{f_{0}}=\frac{1}{2 C_{66}}\left(2 C_{66} \varepsilon_{1}^{(0)}+C_{661} \varepsilon_{1}^{(0)}+C_{662} \varepsilon_{2}^{(0)}+C_{663} \varepsilon_{3}^{(0)}+\right.
$$

Solid-State Sensors, Actuators and Microsystems Workshop Hilton Head Island, South Carolina, June 5-9, 2016 


$$
\left.C_{664} \varepsilon_{4}^{(0)}\right)-\left(\frac{t_{q}^{2} / \sqrt{48}}{\pi C_{66}}\left(C_{165} \frac{\partial \varepsilon_{5}^{(1)}}{\partial x_{1}}+C_{561} \frac{\partial \varepsilon_{1}^{(1)}}{\partial x_{3}}+C_{563} \frac{\partial \varepsilon_{3}^{(1)}}{\partial x_{3}}\right)\right)
$$

where $C_{66}$ is the second order and $C_{p q r}$ is the third order elastic stiffness coefficients, respectively, $\varepsilon_{m}^{(0)}$ and $\varepsilon_{m}^{(1)}$ are the zeroth and first order strains, $t_{q}$ is the thickness of quartz, and $x_{1}, x_{2}$, and $x_{3}$ are the three principal axes in the Cartesian coordinate system.

\section{EXPERIMENT SETUP}

\section{Sensor Fabrication}

$100 \mu \mathrm{m}$ thick, 1 inch diameter AT-cut quartz wafers were cleaned in Nanostrip ${ }^{\circledR}$ for 30 minutes and then $15 \mathrm{~nm}$ chromium (Cr) and $150 \mathrm{~nm}$ gold ( $\mathrm{Au}$ ) was deposited as seed metal layers using an e-beam evaporator. First lithography was done using photoresist SPR 220 to define the etch regions. 12-15 $\mu \mathrm{m}$ Nickel was electroplated that acts as a hard mask for plasma etching. Patterned quartz wafers were then etched in Alcatel AMS 100 inductively coupled plasma reactive ion etcher (ICP-RIE) using Argon and $\mathrm{SF}_{6}$ gases. After completion of etching, the remaining metal mask was removed in aqua regia $\left(\mathrm{HCl}: \mathrm{HNO}_{3}:: 3: 1\right) .20$ $\mathrm{nm} / 150 \mathrm{~nm}$ of $\mathrm{Cr} / \mathrm{Au}$ was deposited using an evaporator, second lithography was done and $\mathrm{Cr} / \mathrm{Au}$ was wet-etched to define an electrode on one side of the resonator. A third lithography was done on the other side of the resonator using LOR5A and SPR 3012. Again, $20 \mathrm{~nm} / 150 \mathrm{~nm}$ of $\mathrm{Cr} / \mathrm{Au}$ was deposited using an evaporator and finally lift off was done using Remover PG to define electrodes. Figure 1 schematically illustrates the process flow. The fabricated quartz sensors were cut into smaller pieces and mounted on a modified ceramic dual in-line package having a $5 \times 5 \mathrm{~mm}^{2}$ square hole machined in the center. RTV 157 silicone epoxy was applied all around the quartz piece to hermetically seal the two sides of the sensor while minimizing any possibility of stressing the quartz as shown in Fig. 2.

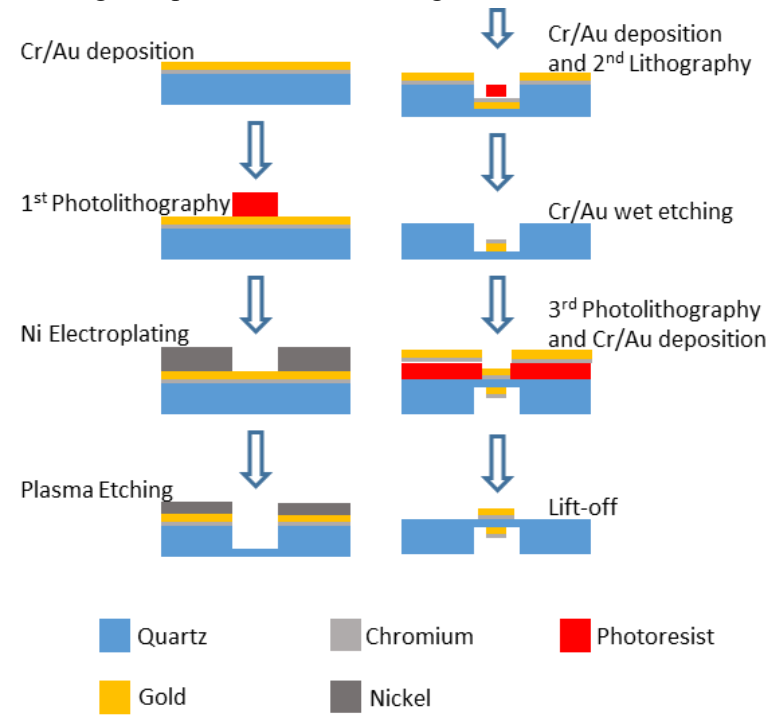

Figure 1: Sensor fabrication process

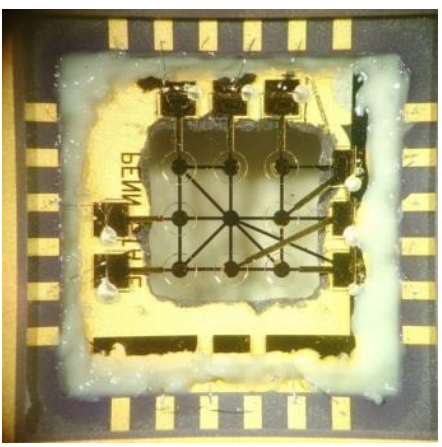

Figure 2: Finished device mounted on the package

\section{Sensor Characterization Setup}

The packaged device in the ceramic package was mounted on a custom made package fixture. This fixture hermetically seals the two sides of the quartz diaphragm with the help of a gasket on one side and an O-ring on the other. Figure 3 shows the mounting of the sensor on the custom fixture in detail. Finally, this fixture is connected to a vacuum system with the help of Swagelok fittings. The two sides of the diaphragm are isolated with the help of an isolation valve and a pressure difference is created by introducing nitrogen on one side of the diaphragm. The pressure is monitored using an MKS Baratron ${ }^{\circledR}$ capacitance manometer and an MKS Cold Cathode Gage. The resonance characteristics of the sensor were monitored with the help of 4294A Agilent Precision Impedance Analyzer. Figure 4 shows a schematic of the test setup.

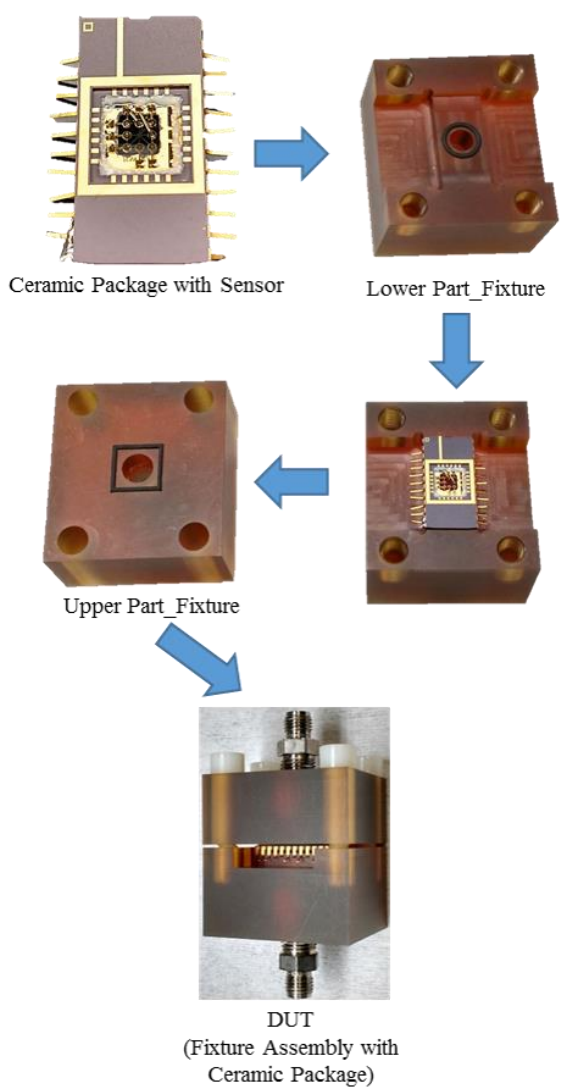

Figure 3: Sensor assembly process 


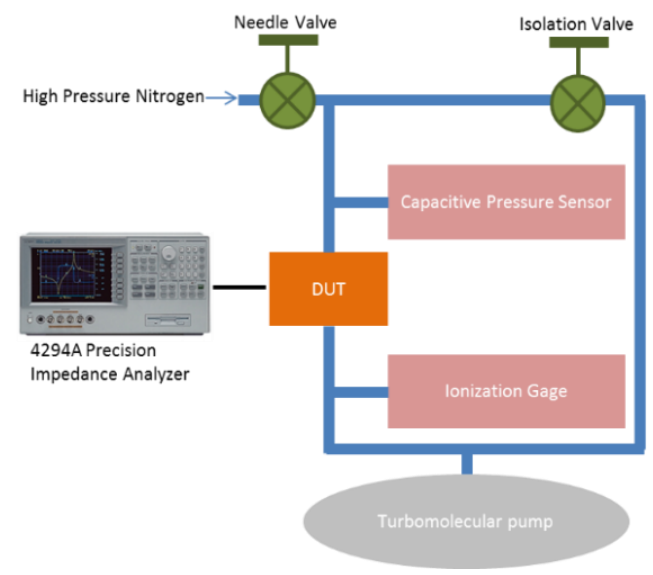

Figure 4: Schematic of test setup

\section{RESULTS AND DISSCUSSIONS}

All pressure sensing experiments were done after bringing both sides of the diaphragm into the high vacuum regime $(\sim 10$ $\mu$ Torr) and then isolating the two sides with the help of the isolation valve. Thus, one side of the diaphragm is constantly under high vacuum whereas the other side is subjected to variable pressure by the introduction $\mathrm{N}_{2}$ gas through a needle valve. The quartz diaphragm is constantly resonated in thickness shear mode with the help of impedance analyzer. Its conductance and susceptance properties were monitored as a function of the applied pressure on one side of the quartz diaphragm. For high resolution and small pressure range of $1-2$ mTorr, the susceptibility of the resonator was monitored at a constant frequency $f_{0}$, set by the unloaded resonance frequency, which was determined by the peak of the conductance maximum as seen in the typical resonance characteristic of an $83.56 \mathrm{MHz}$ resonator in Fig. 5. This method is limited to the frequency range between the two inflexion points in the susceptance curve shown in Fig. 5. Thus for measurements involving larger pressure range of $>1$ Torr, the resonance frequency shift of conductance was monitored as a function of pressure.

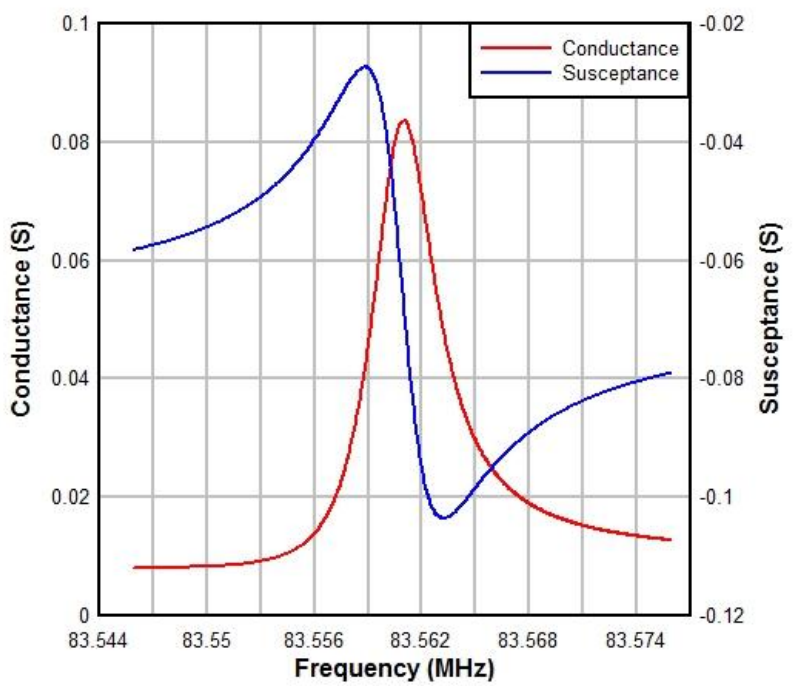

Figure 5: Conductance and susceptance characteristics of an 83.56 MHz AT cut quartz resonator excited in thickness shear mode.

\section{Pressure Sensitivity with Thickness}

Figure 6 shows the dependence of the susceptance of the resonator as a function of applied pressure for two resonators. Both resonators have a diaphragm diameter of $1 \mathrm{~mm}$. However, the thickness of the two resonators is $20 \mu \mathrm{m}$, corresponding to $f_{0}=$ 83.56 MHz and $37 \mu \mathrm{m}$, corresponding to $f_{0}=45.5 \mathrm{MHz}$. From eq. (1) it is clearly evident that as the thickness of the diaphragm is reduced, the peak deflection will increase proportionally. Thus, the strain and strain gradients in the diaphragm are also expected to increase, resulting in a fractional change in the frequency as given by eq. (2). Figure 6 shows the at-resonance susceptibility sensitivity of two devices as a function of the applied pressure. Clearly, a linear dependence of the frequency on applied pressure is observed for both the resonators. The straight lines are the least square fits to the experimentally obtained data and are used to calculate the pressure sensitivity for the two resonators. At the respective resonance frequencies, the slope of the $83.56 \mathrm{MHz}$ resonator was $10.65 \mu \mathrm{S} / \mathrm{mT}$ Torr, whereas for the $45.5 \mathrm{MHz}$ resonator the slope is $3.46 \mu \mathrm{S} / \mathrm{mT}$ Torr. Thus, the slope for $\sim 20 \mu \mathrm{m}$ $(83.56 \mathrm{MHz})$ thick resonator is about three times higher than the slope of $\sim 37 \mu \mathrm{m}(45.5 \mathrm{MHz})$ resonator.

\section{Noise Characteristics and Range of the Resonator}

Resonator noise was experimentally measured to be $38.5 \mu \mathrm{S}$ for the $45 \mathrm{MHz}$ resonator and $25 \mu \mathrm{S}$ for the $83.56 \mathrm{MHz}$ resonator for a bandwidth of $1.35 \mathrm{~Hz}$ (i.e. for $0.74 \mathrm{sec}$ time constant). Based on the sensitivity measurements shown in Fig. 6, this corresponds to a SNR resolution of $11.14 \mathrm{mT}$ Torr for the $45.5 \mathrm{MHz}$ resonator and 2.39 mTorr for the $83.56 \mathrm{MHz}$ resonator. The biggest source of noise could be attributed to the vibrations on the measurement set-up from various sources such as the vacuum pump and floor vibrations and thus could be improved by better damping and isolation of the sensors. The range of linear response for both the 45.5 $\mathrm{MHz}$ and $83.56 \mathrm{MHz}$ resonators was $0-80$ Torr. However, in this larger pressure range, the $83.56 \mathrm{MHz}$ resonator showed only two times higher sensitivity than the $45.5 \mathrm{MHz}$ resonator. The burst differential pressure for the sensor diaphragm for the $83.56 \mathrm{MHz}$ resonator was determined to be around 350 Torr leading to a catastrophic failure of the sensor.

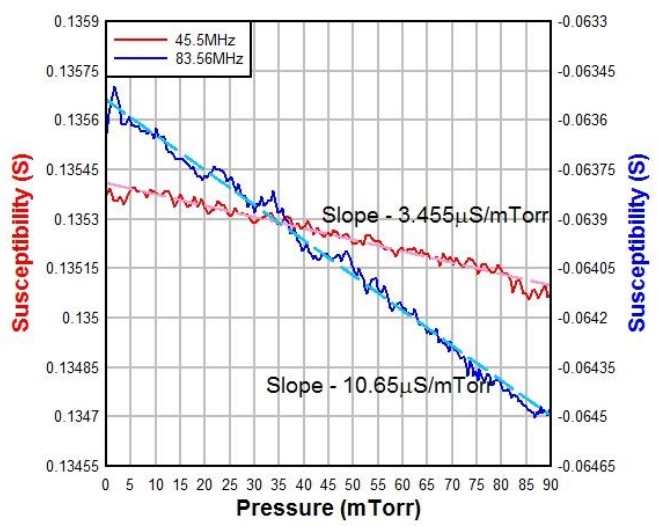

Figure 6: Comparison of at-resonance susceptance versus change in pressure for $20 \mu \mathrm{m}(83.56 \mathrm{MHz})$ and $37 \mu \mathrm{m}(45.5 \mathrm{MHz})$ thick resonators.

\section{Pressure Sensitivity to Direction of Pressure Application}

Figure 7 shows that the quartz pressure sensor response also depends on the side of the diaphragm on which the pressure was 
applied. Similar observations have been reported for in-plane applied stresses, whereby changing the sign of diametric force application from tensile to compressive or vice-versa, resulted in the sign of the change in resonance frequency to reverse [11]. The cause of this behavior is currently under investigation.

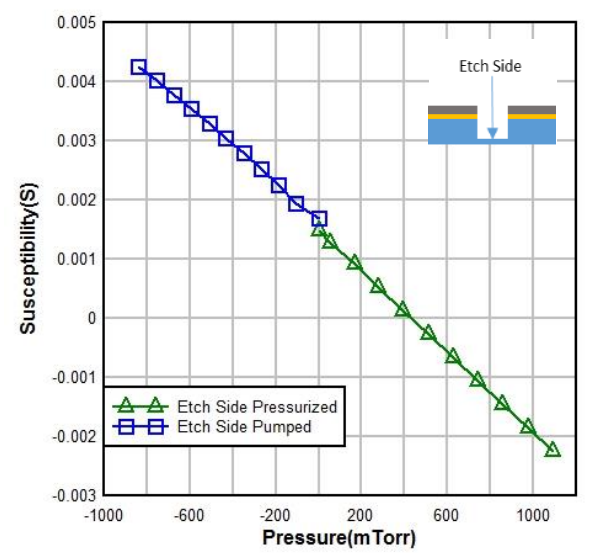

Figure 7: Susceptibility v/s pressure for $83.56 \mathrm{MHz}$ resonator showing bi-directionality (Inset: Showing etch side)

\section{CONCLUSION}

In this report, we have demonstrated a micromachined quartz diaphragm based pressure sensor with a range of operation from 1 mTorr to 80 Torr. A resolution of 11.14 mTorr and 2.39 mTorr for $45.5 \mathrm{MHz}(\sim 37 \mu \mathrm{m}$ thick) and $83.56 \mathrm{MHz}(\sim 20 \mu \mathrm{m}$ thick) resonator has been demonstrated. The range of operation has also been explored where sensors show a linear response in the $0-80$ Torr pressure range with the $83.56 \mathrm{MHz}$ resonator having higher sensitivity compared to the $45.5 \mathrm{MHz}$ resonator. Bi-directionality of the pressure sensor has also been shown. The sensitivity is shown to be a function of thickness and thus can be tuned by changing the thickness of the quartz diaphragm. A theoretical model to explain the performance of the quartz resonator as a function of applied pressure is currently under development and will be presented in future.

\section{ACKNOWLEDGEMENTS}

This work was supported by MKS Instruments, Inc. The use of facilities at the PSU site of the NSF National Nanotechnology Infrastructure Network (NNIN) under Agreement 0335765 is also acknowledged.

\section{REFERENCES}

[1] S. Wilfert and C. Edelmann, "Miniaturized vacuum gauges," J. Vac. Sci. Technol. A Vacuum, Surfaces, Film., vol. 22, no. 2, p. 309, 2004.

[2] X. Wang, C. Liu, Z. Zhang, S. Liu, and X. Luo, "A micro-machined Pirani gauge for vacuum measurement of ultra-small sized vacuum packaging," Sensors Actuators, A Phys., vol. 161, no. 1-2, pp. 108-113, 2010.

[3] F. T. Zhang, Z. Tang, J. Yu, and R. C. Jin, "A micro-pirani vacuum gauge based on micro-hotplate technology," Sensors Actuators, A Phys., vol. 126, no. 2, pp. 300-305, 2006.

[4] F. Völklein and A. Meier, "Microstructured vacuum gauges and their future perspectives," Vacuum, vol. 82, no. 4, pp. 420-430, 2007.

[5] J. Dobrott and R. Oman, "Ionization Gauge Using a SiC pn Junction Electron Emitter," J. Vac. Sci. Technol., vol. 7, no. 1970, p. 214, 1970.
[6] D. Nicolaescu, V. Filip, and F. Okuyama, "Study of the inverted-magnetron cold emission microelectronic vacuum gauge," Ultramicroscopy, vol. 73, no. 1-4, pp. 129-137, 1998.

[7] D. Blechschmidt, "A miniature extractor gauge for the UHV," J. Vac. Sci. Technol., vol. 11, no. 6, p. 1160, 1974.

[8] W. Eaton and J. Smith, "Micromachined pressure sensors: review and recent developments," Smart Mater. Struct., vol. 6, pp. 530-539, 1997.

[9] S. Tadigadapa and S. Massoud-Ansari, "Applications of High-Performance MEMS Pressure Sensors Based on Dissolved Wafer Process," Sensors (Peterborough, NH).

[10] Y. Zhang and K. D. Wise, "Performance of NonPlanar Silicon Diaphragms under Large Deflections," J. Microelectromechanical Syst., vol. 3, no. 2, pp. 59-68, 1994.

[11] J. M. Ratajski, "The force sensitivity of AT Cut Quartz Crystals," pp. 33-49.

[12] P. C. Y. Lee, Y. S. Wang, and X. Markenscoff, "Nonlinear effects of initial bending on the vibrations of crystal plates," pp. 90-96, 1975.

[13] E. P. EERNISSE and R. B. WIGGINS, "Review of thickness-shear mode quartz resonator sensors for temperature and pressure," IEEE Sens. J., vol. 1, no. 1, pp. 79-87, 2001.

[14] M. D. Giovanni, Flat and Corrugated Diaphragm Design Handbook, First ed. New York: Marcel Dekker, 1982.

\section{CONTACT}

S. Tadigadapa: Tel. (814) 865 2730, sat10@psu.edu 